\title{
Studies on Manganese
}

\author{
III. THE BIOLOGICAL HALF-LIFE OF RADIOMANGANESE IN \\ MAN AND FACTORS WHICH AFFECT THIS HALF-LIFE
}

John P. Mahoney and Walter J. SMall

From the Research Laboratory, Carney Hospital, Boston, Massachusetts

\begin{abstract}
A вSTRACT The biological half-life of manganese and some factors influencing it have been studied in man. The disappearance of manganese from the body in normal subjects is described by a curve having two exponential components. An average of $70 \%$ of the injected material was eliminated by the "slow" pathway. The half-time characterizing this component showed a small variation in normal subjects and had an average value of 39 days. The half-time for the "fast" component also showed a small variation and had an average value of 4 days.

In a normal subject presumed to have a low manganese intake due to a voluntary low caloric intake, the percentage eliminated by the slow pathway increased to $84 \%$ and the half-time characterizing the pathway increased to 90 days. The half-time of the "fast" component was the same as for the normal group. 2 months after initiation of the study in this subject, a large "flushing" dose of manganese markedly increased the elimination rate which was described by a single exponential curve.
\end{abstract}

A mildly iron-deficient subject showed a marked decrease in the percentage of manganese eliminated by the "slow" pathway accompanied by a less dramatic decrease in the half-time characterizing this pathway. Oral iron therapy, which corrected the mild anemia, caused a decrease in the elimination rate and the altered curve was described by a single exponential component.

Preloading two subjects with manganese resulted in a great decrease in the fraction eliminated

Received for publication 22 June 1967 and in revised form 13 October 1967. by the "slow" pathway with less effect on the half-time. The subject with the largest preloading dose showed no "slow" component at all.

Observations on the red cells of some of these subjects showed that a small but definite fraction was incorporated into the erythrocytes.

In the mildly iron-deficient subject, our observations suggest an interrelationship between manganese and iron metabolism.

\section{INTRODUCTION}

Our laboratory has had a long-standing interest in the metabolism of manganese since there is abundant evidence that this element is an essential trace metal. This has led us to carry out studies on the disappearance of injected radiomanganese from the plasma and its incorporation into erythrocytes as a manganese porphyrin. In order to pursue these studies further we performed calculations of the total body radiation which would result from the administration of ${ }^{54} \mathrm{Mn}$ in appropriate doses. The calculations of the total body radiation were based upon a biological half-life of manganese of 10-15 days. The assumption for the biological half-life was based upon data reported by Borg and Cotzias (1) from studies on rats, and also upon the statement by the International Commission on Radiation Protection (2), that the biological half-life of manganese in humans was 17 days.

Since our proposed studies would involve observations for a period of at least 100 days, and in some cases longer (the life span of the erythrocyte), it was essential that we use an isotope with 
a long physical half-life. Because of the quantity of radioactivity which we planned to administer and because of the long physical half-life of ${ }^{54} \mathrm{Mn}$ (291 days), we decided to study the true biological half-life in man. This would profoundly affect the effective half-life and, therefore, the total body radiation.

The purpose of this report is to describe our observations on the biological half-life of intravenously injected ${ }^{54} \mathrm{Mn}$ and the effect on this half-life of loading the body with inert manganese. The data to be presented indicate that our concern over the published biological half-life was indeed well founded.

\section{METHODS}

The studies were carried out in the low background "Iron Room" facility at Boston University Medical School ${ }^{1}$ with which Carney Hospital is affiliated. This facility, which has been described elsewhere (3), is uniquely suited for resolution of the question at issue. The facility permitted studies of the whole body ${ }^{54} \mathrm{Mn}$ for in excess of 90 days with good statistical reliability even though the injected dose of ${ }^{54} \mathrm{Mn}$ was only $2.5 \mu \mathrm{c}$ in all subjects studied.

The technique was to scan by moving the total body under two fixed $8 \times 4$ inch Harshaw thallium-activated sodium iodide crystals (The Harshaw Chemical Co., Cleveland, Ohio) on a motor-driven bed. The total body was scanned twice for each observation, once in the supine position and then immediately in the prone position.

The ${ }^{54} \mathrm{Mn}$ used for the injections was cyclotron produced by Nuclear Science and Engineering Co., Pittsburgh, Pa., and supplied as a sterile pyrogen-free solution by Isoserve Inc., Cambridge, Mass. It was a carrier-free solution of ${ }^{54} \mathrm{MnCl}_{2}$ made isotonic with saline. The radiopurity of the isotope was certified by Isoserve on the basis of gamma ray spectrometry. Before injection we rechecked the gamma spectrum at the Boston University facility, utilizing the TMC 400 channel analyzer customarily employed to collect and transfer data from the whole body scanner. This assay verified that the isotope was $99 \%+{ }^{54} \mathrm{Mn}$.

For all assays of radioactivity spectrometer settings were selected to integrate gamma energies between $740-$ $940 \mathrm{kev}$, thus the entire $840 \mathrm{kev}$ gamma peak of ${ }^{54} \mathrm{Mn}$ was included. All data were normalized to the maximum integrated peak activity observed in each individual. Interestingly this peak activity was observed 1-2 days after the injection which we assume was due to delay in distribution among the various organs of the body.

In an effort to account for the total radioactivity injected, excreta (urine and stools) were collected and assayed in the same facility. ${ }^{54} \mathrm{Mn}$ standard solutions were made up to appropriate volumes geometrically equivalent to the urine and stool specimens. Counting efficiency for

\footnotetext{
1 Courtesy of Dr. Belton A. Burrows.
}

stool and urine specimens and their standards in the facility was nearly $100 \%$, while for the whole body the efficiency was $6.5 \%$.

It was also of interest to measure the radioactivity in the blood. For this aspect of the study, blood was collected in double plastic bags ${ }^{2}$ under standard blood bank conditions and was separated into its red cell and plasma components by centrifugation at $1190 \mathrm{~g}$ in an International PR-2 refrigerated centrifuge. After assay for radioactivity, the cells and plasma were reconstituted and immediately reinfused into the subjects.

All subjects were informed that they were receiving a radioactive isotope as an experimental procedure and gave their informed consent. The protocol was approved by the Committee on the Use of Humans as Experimental Subjects of the Carney Hospital and Boston University Medical School.

Six volunteer subjects from hospital personnel were studied. The age range of the volunteers was from 25$45 \mathrm{yr}$ and both males and females were included. For purposes of discussion, the subjects are presented and discussed separately.

Subject (J.M.) a male, had voluntarily been on a diet estimated at $800 \mathrm{cal} / \mathrm{day}$ for 6 months before the initiation of the study in order to lose weight. This weight loss regimen continued throughout the interval covered by the study. A weight loss of $15 \mathrm{lb}$. had been realized before the study and a further weight loss of $10 \mathrm{lb}$. was realized during the course of this study. Otherwise the physical and laboratory examinations, which were part of the protocol, were unremarkable.

60 days after the initiation of the study, this subject began to ingest $800 \mathrm{mg}$ of manganese per day as a solution of manganese chloride. This regimen was continued for 35 days. After 25 days on this manganese regimen, the original manganese activity had dropped to $20 \%$ of the original value which permitted the initiation of a repeat study in this volunteer. The objective of the second study was to confirm the proposition that the ingestion of manganese by subject (W.S.), who was preloaded with manganese, was in fact responsible for the observed rapid disappearance of the metal. The data on subject (J.M.) are also presented and discussed separately.

All of the data reported are expressed as a percentage of the maximum activity observed in counting the total body in the prone and supine position. Usually maximum activity was observed on day 2 or 3 . The $2.5 \mu \mathrm{c}$ of ${ }^{54} \mathrm{Mn}$ injected resulted in an average maximum for total body counts of $35,300 \mathrm{cpm}$ for the supine position and 29,500 $\mathrm{cpm}$ for the prone position. Counting time in all cases was $2 \mathrm{~min}$. The reference $100 \%$ points were different for the two counting positions and were also different from individual to individual. The difference in the counting rate from individual to individual is explained by differences in weight and body size which caused variations in geometry and the amount of radiation absorbed by intervening tissue. The counting rate difference for the supine position as compared to the prone position is probably related to the distribution of isotope throughout the body.

\footnotetext{
2 Fenwal Blood Pack Unit, Code JA-25.
} 


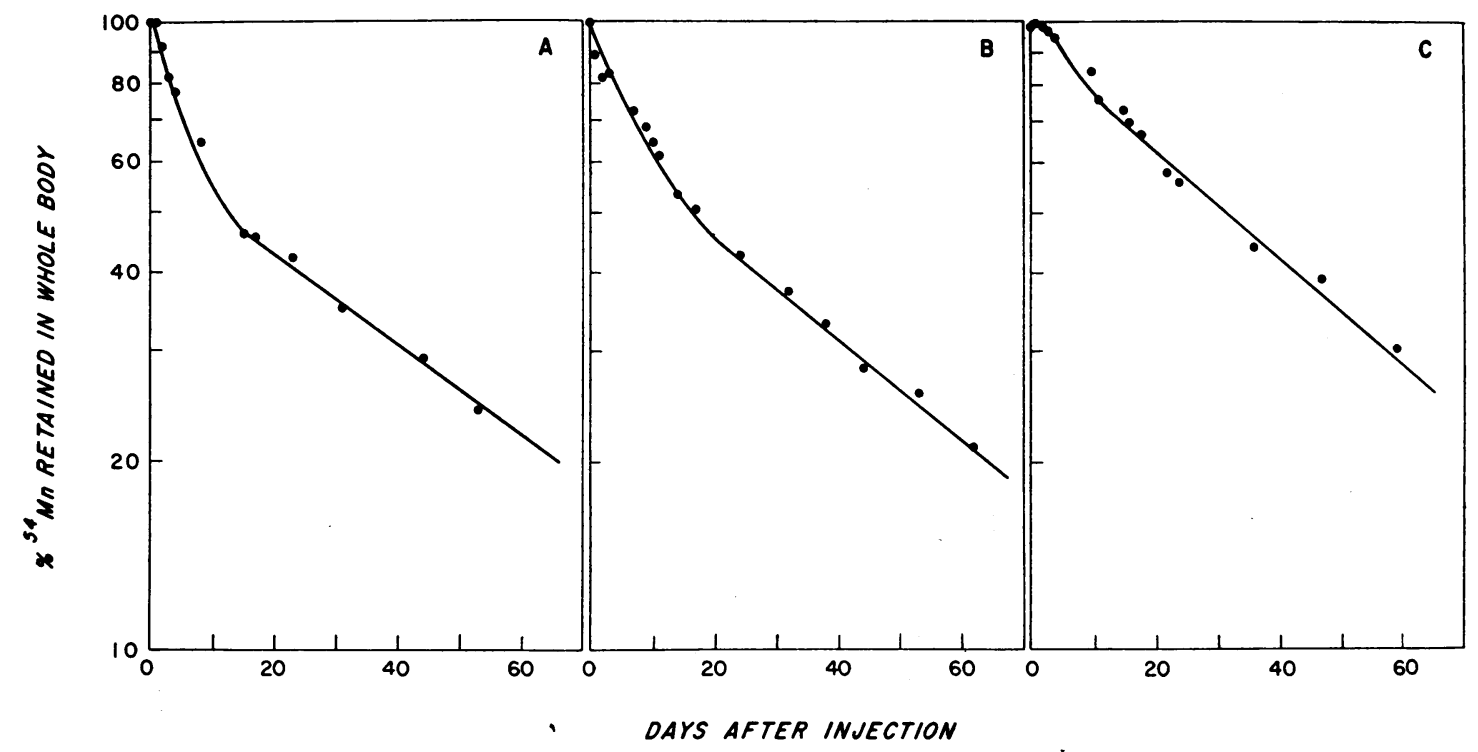

FIGURE 1 Disappearance of manganese from normal subjects. The points are the average value for supine and prone positions. Curve drawn as best fit by eye. $A$ is subject H.M., $B$ is subject M.M., and $C$ is subject $C . H$.

In all cases the curves are based on points derived by averaging the data from the two counting positions.

\section{RESULTS}

The data for the three normal subjects are presented in Fig. 1 and are plotted on semilogarithmic paper with time after injection on the abscissa and per cent remaining in the total body on the ordinate. Analysis of the curves disclosed that the observed data can be analytically described by two exponential components. We accomplished separation into components by extropolating the longlived component to zero time. We characterized the shorter-lived component by subtracting the extropolated long-lived component from the total curve. In all cases the resulting difference curve which characterized the short-lived component was well represented by a straight line when replotted on semilogarithmic paper.

The analysis of the data for subject M.M. is presented in Fig. 2 to illustrate the adequacy of the two component model in accounting for the observed data. The calculated difference curve is clearly linear even at the last observed difference point which was $4 \%$.

The results obtained by this method of data analysis for the three subjects in the normal group are presented in Table $I$.

The half-lives of disappearance for the "slow" component of the normal group are in good agreement with each other. These three subjects had a mean half-life for this "slow" component of 39 days. However, there is a difference in the percentage of the manganese which disappears from the body by the pathway characterized by this "slow" component in these subjects. Two of them were in good agreement at $60-65 \%$ but the third, (C.H.), at $91 \%$ was significantly greater and, therefore, indicated that caution is warranted before conclusions are drawn. We have no explanation at this time for this large and significant variation.

The "fast" component for the normal group also showed good agreement with respect to the apparent half-time characterizing the pathway. The three values are quite close to each other and if averaged give a value of 3.9 days.

The data on subject S.M., in whom mild irondeficiency was discovered, are presented in Fig. 3. Analysis of the curve to day 50 , i.e. before initiation of iron therapy, shows that the "slow" component pathway processed $52 \%$ of the activity when extropolated to zero time, and this component was characterized by an apparent half-time of 37 days. The "fast" component was characterized by an apparent half-time of 5 days.

Except for the decrease in the fraction of manganese eliminated by the "slow" component path- 


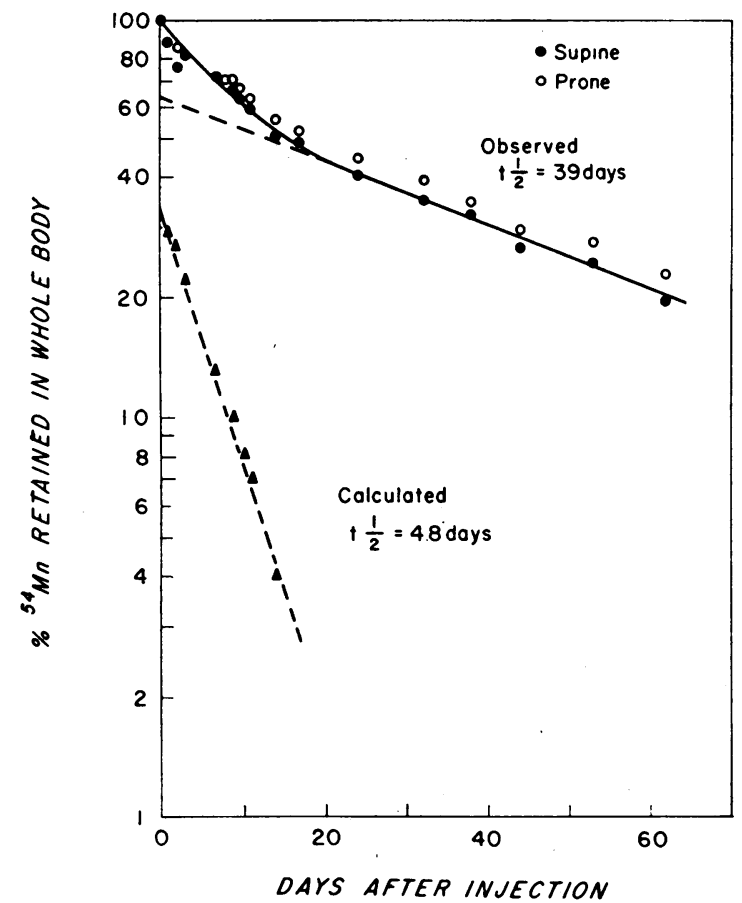

FIGURE 2 Analysis of disappearance curve for subject M.M. into components. Open points are observed data for prone position. Solid points are observed data for supine position. Solid triangles calculated by difference.

way, these values agree well with those in Table I for the normal group. Of greater interest are the data collected after initiation of the oral iron therapy regimen on day 50 .

The extropolation of the curve is shown as the dashed line, while the observed data are shown as solid lines. The sharp change in slope of the curve for the observed data indicates that there was a decrease in the rate of elimination, and that this altered metabolism is characterized by a single exponential function. The apparent half-time of

TABLE I

Disappearance of i.v. Injected ${ }^{54} \mathrm{MnCl}_{2}$ from Whole Body-Normal Group

\begin{tabular}{|c|c|c|c|c|}
\hline \multirow[b]{2}{*}{ Subject } & \multicolumn{2}{|c|}{ Component 1} & \multicolumn{2}{|c|}{ Component 2} \\
\hline & $\begin{array}{c}\% \text { of } \\
\text { maximum } \\
\text { observed } \\
\text { activity }\end{array}$ & $t_{1}$ & $\begin{array}{c}\% \text { of } \\
\text { maximum } \\
\text { observed } \\
\text { activity }\end{array}$ & $t_{1}$ \\
\hline & & days & & days \\
\hline C.H. & 9 & 3.5 & 91 & 36.5 \\
\hline M.M. & 35 & 4.8 & 65 & 39 \\
\hline H.M. & 40 & 3.5 & 60 & 41 \\
\hline
\end{tabular}

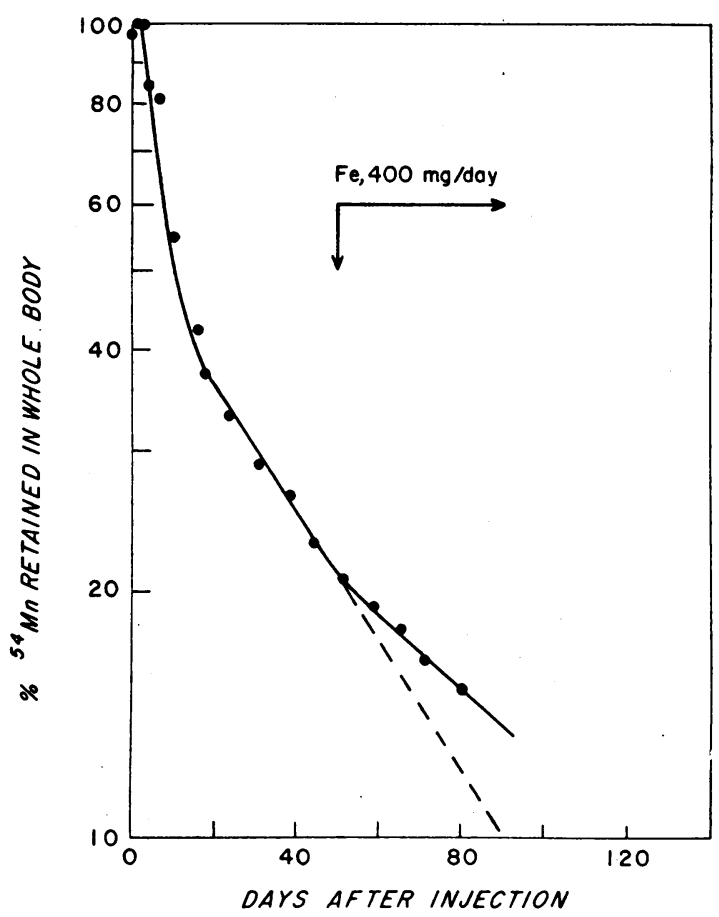

FIGURE 3 Disappearance curve for mildly iron deficient subject S.M. The points are average values for supine and prone positions. Curve drawn as best fit by eye. Dotted curve is extrapolation of line before initiation of iron therapy. Solid line is observed data after initiation of iron therapy.

the new curve is 66 days. As mentioned, during the period the subject's hemoglobin rose to 12.4 $\mathrm{g} / 100 \mathrm{ml}$. The interesting implication is that manganese was involved in the increased hemopoiesis, perhaps as a cofactor. This implication will be the subject of subsequent study.

It seemed important to study the effect of manganese intake on the rate of disappearance both qualitatively and quantitatively. One subject, W.S., was preloaded for 10 days before the experiment by ingesting $300 \mathrm{mg}$ of manganese per day as a solution of manganese chloride. This regimen continued throughout the experiment. Since the reported values for total body manganese are 10-20 mg (4), this represented a daily intake at least 15 times the total body content.

The data on this subject are presented in Fig. 4. Analysis of Fig. 4 again revealed a two component system. $51 \%$ of the injected ${ }^{54} \mathrm{MnCl}_{2}$ disappeared by the "slow" component which in this case was characterized by an apparent half-time of 33 days. The remaining $49 \%$ disappeared as the 


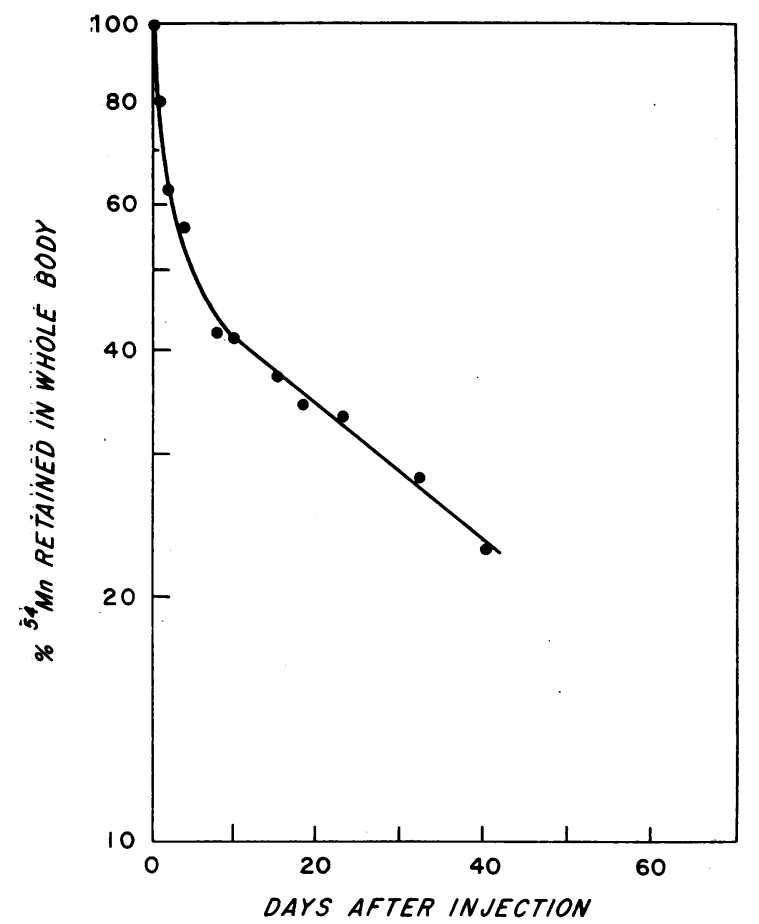

FIGURE 4 Disappearance curve for subject W.S. preloaded with manganese. Data points are average for the two counting positions. Line is best fit by eye. The subject was preloaded for 10 days before injection of ${ }^{54} \mathrm{Mn}$ by $300 \mathrm{mg} /$ day of stable manganese by oral route.

"fast" component characterized by an apparent half-time of 1.5 days.

Comparing the data on the preloaded subject with the normal group reveals a slight decrease in both the percentage disappearing by the "slow" component and the apparent half-time characterizing this component. The major effect is the threefold decrease in the apparent half-time characterizing the "fast" component. The over-all effect of the changes induced in this preloaded subject was to decrease the time required for the manganese in the body to reach $50 \%$ of its original value from the 21 day average observed in the normal group to 7 days. This is in qualitative agreement with observations reported by Britton and Cotzias (5) in mice, but it should be stressed that quantitative comparisons are not valid because they measured the time required for $98 \%$ of the manganese to disappear rather than the time to reach $50 \%$ of the original manganese activity.

To expand the observations in subject W.S., an experiment on the effect of intake of man- ganese was performed on the volunteer J.M., who was independently on the strict weight loss dietary regimen of approximately $800 \mathrm{cal} /$ day. In the first study on this subject, the same protocol as followed for the normal group was followed with the exception that the $800 \mathrm{cal} /$ day diet was continued throughout the study. After 60 days on this regimen, a program of ingestion of $800 \mathrm{mg}$ of manganese per day as a manganese chloride solution was initiated in an effort to "flush" the radiomanganese from the body. Observations were continued during the 25 day course of this manganese regimen. The data are presented in Fig. 5. Again, the points are the average for the two counting positions.

Two components are again observed. Considering the data during the first 60 days, the "slow" component was the pathway for $84 \%$ of the injected ${ }^{54} \mathrm{MnCl}_{2}$ and it was characterized in this case by an apparent half-time of 92 days. The "fast" component had an apparent half-time of 3.5 days. In this case the time required to reach $50 \%$

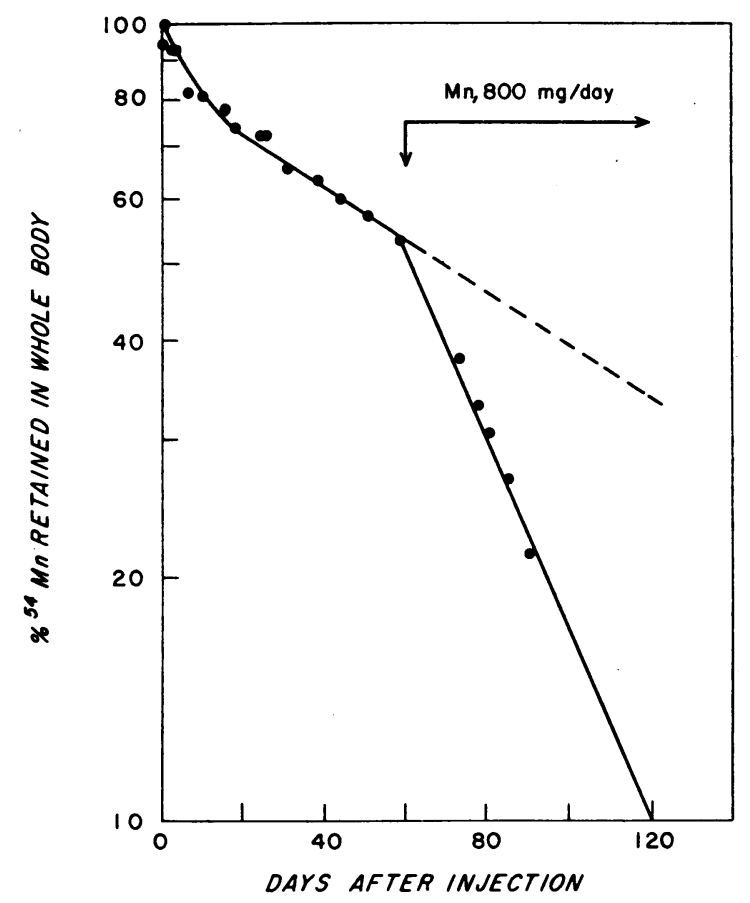

FIGURE 5 Disappearance curve for subject J.M. on low caloric intake. Data points are the average for the two counting positions. Curve is best fit by eye. Dotted line is extrapolation of data before initiation of "flushing" dose. Solid line is the observed data after initiation of flushing dose. 


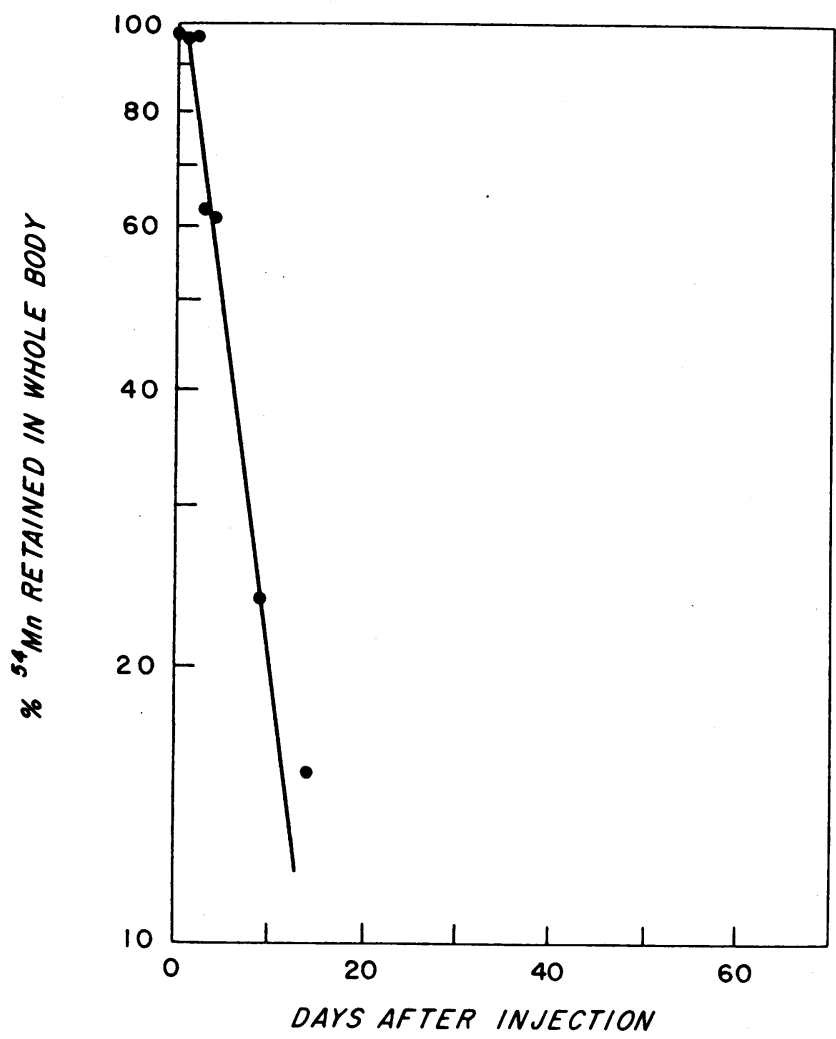

Figure 6 Disappearance curve for subject J.M. after preloading with manganese. Data points are the average for the two counting positions. Curve is best fit by eye. of the original manganese activity value was 70 days. This large difference, compared with the average value of 21 days in the normal group, is accounted for both by the striking increase in the apparent half-time characterizing the "slow" component as well as the increase in the fraction of total manganese eliminated by this pathway.

The data collected after initiation of the manganese "flushing" dose fall considerably below the extropolated line, shown as a dashed extension of the curve in Fig. 5, indicating a dramatic increase in the rate of excretion of the original ${ }^{54} \mathrm{MnCl}_{2}$. The data for this 25-day interval are well represented by a straight line on the semilog plot and, therefore, the increased excretion is a single exponential function which can be characterized by an apparent half-time of 28 days.

At day 85 of the original experiment, a new study was begun on this same volunteer. A fresh injection of $2.5 \mu \mathrm{c}$ of ${ }^{54} \mathrm{MnCl}_{2}$ was administered. The $800 \mathrm{mg}$ of manganese per day regimen was continued throughout this new study. We calculated total body retention for the second manganese injection by subtracting the amount of activity due to the first injection on the assumption that the line observed between day 60 and 85 of the first study could be validly extropolated. These data are presented in Fig. 6 . The striking feature of the data is the elimination of the slow component. The time to reach $50 \%$ of the injected manganese activity in this case was 5 days, and since there is no evidence for "slow" component, this also is the apparent half-time for the "fast" component.

The data in this study are particularly interesting since this volunteer was his own control both for a presumed manganese deficient intake through the first 60 days of the first experiment and for the flushing effect of manganese intake from day $60-85$ of the study.

\section{Recovery of injected ${ }^{54} \mathrm{Mn}$ and excretory routes}

The availability of subjects who had been injected with ${ }^{54} \mathrm{Mn}$ and sensitive equipment for counting their excreta presented the opportunity to examine the routes of excretion of manganese in man and permitted us to attempt a balance study 
in these subjects. The per cent of the injected radioactivity retained in the whole body plus the cumulative per cent excreted (urine plus stool) should account for the total injected radioactivity within the experimental error.

All stools and urine excreted during the early period of the experiment were collected. Samples were combined and counted as the facility became available, hence the day on which the data were collected are not the same for the three individuals.

When it became apparent that none of the material was excreted in the urine and that good correlation was observed between body retention and excretion, this part of the experiment was terminated.

These studies are reported for three of the volunteers in Table II. J.M. is the subject on the $800 \mathrm{cal} /$ day diet and the data presented represent total stool and urine collections and total body retention during the first 22 days, i.e., when the "slow" component of the disappearance curve had the longest apparent half-time (92 days).

S.M. is the female subject with the slight iron deficiency anemia. The data in Table II were collected before initiation of the oral iron therapeutic regimen, i.e., when the "slow" component had the shortest apparent half-time (37 days). M.M. is the normal female subject.

As would be anticipated from the total body retention data, a greater amount of the injected activity appeared in the feces in the first 10 or
15 days of the study. The "fast" component makes the major contribution to excreted activity during this interval. This is reflected in the data since J.M. who had the smallest percentage of injected manganese eliminated, also had the slowest excretion rate. Examination of the data shows that in the first 5 days an average excretion rate of about $1 \% /$ day was eliminated by J.M. (5.9\% total in the first 5 days). In the next 6 days, only an additional total of $2.4 \%$ was excreted, an average excretion rate of roughly $0.5 \% /$ day, bringing the accumulated total excreted in the stool to $7.9 \%$. For the next two intervals, the average rate for J.M. returned to about $1 \%$ /day.

Subjects S.M. and M.M. also showed an increased excretion rate during the first 5-day interval. Thereafter both of these subjects showed an average excretion rate of about $2 \%$ /day. Both this agreement between the rates and the fact that they are about twice the rate in J.M. would be expected from the total body retention data.

The failure to account for $100 \%$ of injected radioactivity can be attributed to loss of excreta in collection and difference between counting efficiencies for excreta as compared with total body count.

\section{Radioactivity in blood}

The fraction of injected ${ }^{54} \mathrm{MnCl}_{2}$ which entered the erythrocytes was determined in four of the volunteers. Two data points each on subject J.M.,

TABLE II

Recovery of Radioactivity after Injected ${ }^{54} \mathrm{MnCl}_{2}$

\begin{tabular}{|c|c|c|c|c|c|c|c|c|c|c|c|}
\hline \multirow[b]{3}{*}{ Time, days........... } & \multicolumn{11}{|c|}{ Subject } \\
\hline & \multicolumn{4}{|c|}{ J.M. } & \multicolumn{4}{|c|}{ S.M. } & \multicolumn{3}{|c|}{ M.M. } \\
\hline & $1-5$ & $5-11$ & $11-16$ & $16-22$ & 5 & 11 & 15 & 18 & 4 & 10 & 15 \\
\hline $\begin{array}{l}\text { Remaining in body. } \\
\% \text { of inj. dose }\end{array}$ & 86 & 78.8 & 76.5 & 73.0 & 79.0 & 52.0 & 43.5 & 37.8 & 82.0 & 64.6 & 51.5 \\
\hline $\begin{array}{l}\text { Cumulative excretion } \\
\text { in urine }\end{array}$ & 0.02 & 0 & 0 & 0 & 0 & 0 & 0 & 0 & 0 & 0 & 0 \\
\hline $\begin{array}{l}\text { Cumulative excre- } \\
\text { tion in stool }\end{array}$ & 5.5 & 7.9 & 13.7 & 18.4 & 37.2 & 43.3 & 54.1 & 59.3 & 8.9 & 23.4 & 32.0 \\
\hline $\begin{array}{l}\text { Sum of }{ }^{54} \mathrm{Mn} \text { re- } \\
\text { maining in body } \\
\text { and total }{ }^{54} \mathrm{Mn} \\
\text { excreted }\end{array}$ & 91.5 & 86.7 & 90.2 & 91.4 & 116.2 & 95.3 & 97.6 & 97.1 & 90.9 & 88.0 & 84.0 \\
\hline
\end{tabular}


days 18 and 86, and S.M., days 44 and 86, were taken as well as one each on H.M., day 17, and manganese preloaded W.S., day 10 . We obtained each point by withdrawing $500 \mathrm{ml}$ of blood, separating the plasma and erythrocytes by centrifugation, assaying the radioactivity, reconstituting the blood, and reinfusing the blood into the donor. The data are presented in Table III.

The fraction of radioactivity which appeared in the red cells was quite small, but nevertheless of interest. The one normal in this study (H.M.) incorporated twice as large a fraction as any of the other volunteers. The subject on low calorie intake (J.M.), the manganese preloaded subject (W.S.), and the subject with mild iron deficiency (S.M.), all incorporated about the same small fraction of injected ${ }^{54} \mathrm{Mn}$ into their red cells.

Considering subject J.M., it is apparent that the "flushing" dose of manganese, which was initiated on day 60 , did not exchange with the radioactivity in his red cells since the fraction in the red cells was the same on day 86 as it was on day 18 . On the other hand, S.M. showed a significant decrease in the fraction of ${ }^{54} \mathrm{Mn}$ bound to her red cells after the initiation of the oral iron regimen on day 50 as shown by the decreased fraction on day 86 as compared to day 44 .

The high counting efficiency of the facility permits us to have good confidence in reaching conclusions even though these fractions are quite small. For example for the case of S.M., the packed cells for day 86 had an observed counting rate of $280 \mathrm{cpm}$ above background and counting time was $20 \mathrm{~min}$. Average background counting rate was $75 \mathrm{cpm}$. The expected standard deviation due to counting statistics for $280 \mathrm{cpm}$ above back-

TABLE III

${ }^{54} \mathrm{Mn}$ in Blood after Intravenous Injection

\begin{tabular}{cccc}
\hline & $\begin{array}{c}\% \text { of } \\
\text { original } \\
\text { 34Mn in } \\
\text { total plasma }\end{array}$ & $\begin{array}{c}\text { \% of original } \\
\text { s4Mn in total } \\
\text { erythrocyte pool }\end{array}$ & $\begin{array}{c}\text { Days after } \\
\text { injection }\end{array}$ \\
\hline J.M. & 0 & 0.064 & 18 \\
J.M. & 0 & 0.067 & 86 \\
S.M. & 0 & 0.048 & 44 \\
S.M. & 0 & 0.035 & 86 \\
H.M. & 0 & 0.14 & 17 \\
W.S. & 0 & 0.057 & 10 \\
\hline
\end{tabular}

ground with these counting times is less than $4 \mathrm{cpm}$.

\section{DISCUSSION}

${ }^{54} \mathrm{MnCl}_{2}$ disappearance data from the total body

The observed data on manganese disappearance from the whole body eliminate the possibility that the biological half-life of manganese can be described by a simple constant. They also indicate that the biological half-life is influenced by the intake of manganese. It may also be influenced by other factors, possibly the state of iron stores or hemoglobin concentration. Probably other factors are involved which require further study.

The data presented indicate that there are two components to the disappearance of manganese from the total body in normal subjects. For the "slow" component the apparent half-time is about 39 days and for the "fast" component it is about 4 days. There is good agreement on the values for these half-times among the three subjects in this normal group. However, the per cent of the injected manganese which was eliminated by the respective pathways was widely different. One of the individuals in the normal group, C.H., showed $90 \%$ disappearance by the slow component compared to 60 and $65 \%$ for the other two normal volunteers.

Britton and Cotzias (5), in a recently published study in mice, found that the fraction of injected manganese eliminated by the "slow" component increased from a value of $35 \%$ for the mice which were on a low manganese intake for the shortest time, 5 days, to about $95 \%$ for the group on low manganese prefeeding for 26 days.

The value for the apparent half-time of the "slow" component observed in their study was affected to a lesser extent by manganese intake and decreased from 50 to 35 days as the interval of prefeeding low doses of manganese increased.

The data for the normal group in our study cannot be strictly compared to the mice data of Britton and Cotzias (5) because of the difference in experimental design. However, it is interesting that our human subjects on a normal diet show a fraction eliminated by the "slow" component approximately twice as great as that shown for the mice group presumably closest to normal, i.e., 
those on the prefeeding regimen for the shortest time. In subject C.H., in fact, the "slow" component was similar to the mice group prefed on the low manganese regimen for the longest interval. Also, in man on a normal diet the apparent half-time characterizing the slow component is fairly constant at about 39 days, while the study in mice showed a slight decrease from 50 to 35 days as the low manganese regimen increased from 5 to 26 days.

The implication in the Britton and Cotzias (5) study is that as the amount of manganese eliminated from the body by the "slow" component pathway increased, there was a decrease in the apparent half-time characterizing the "slow" component. In other words, in mice, the fraction eliminated by the slow pathway is inversely related to the half-time characterizing the pathway. In our normal group one subject showed a large increase in percentage eliminated by the "slow" component pathway but the was no change in the apparent half-time characterizing the "slow" component pathway.

The more germane comparison between the two studies is obtained from the data on J.M., in the first 60-day interval, whose low caloric intake we assumed to be low in manganese. In this case the fraction eliminated by the "slow" component greatly increased but the apparent half-time characterizing it also increased. In the studies reported by Britton and Cotzias (5), the fraction processed by the "slow" component increased to $95 \%$ in the mice on the low manganese prefeeding for the longest interval, which agrees with our observations. On the other hand, in the mice study the apparent half-time characterizing the pathway decreased from 50 to 35 days, whereas in our study the apparent half-time increased.

Loading the body with stable manganese by the oral route had a profound effect on the biological half-life of the injected manganese isotope in man. This effect appeared to be qualitatively different than was the case in the Britton and Cotzias (5) study. A large increase in the rate of elimination of radiomanganese was indeed noted in J.M. between day 60 and 85 as a consequence of increased manganese intake. However, the increased elimination was characterized by a curve having a single component rather than the two component curve observed in mice. This difference may be due to the fact that in our study, 60 days elapsed before initiation of "flushing" efforts while in the mice study the interval was considerably shorter, 16 days. Undoubtedly other factors are involved but it is difficult to assess factors such as intake of inert manganese per unit weight in the mice study from the published data. Differences between the species may also be significant.

Effect of preloading with stable manganese on biological half-life of radiomanganese

Two studies in this area are presented. One subject (W.S.) was loaded by the oral route with $300 \mathrm{mg}$ of $\mathrm{Mn}$ per day for 10 days before injection of ${ }^{54} \mathrm{Mn}$. This manganese intake continued throughout the study. The other subject (J.M.) served as his own control. After the flushing study, discussed in the preceding secion, the $800 \mathrm{mg}$ of $\mathrm{Mn}$ per day regimen was continued and a new injection of ${ }^{54} \mathrm{MnCl}_{2}$ made 86 days after J.M. began the original study. The second injection of ${ }^{54} \mathrm{Mn}$ was therefore preceded by a 26 day preloading period.

For both subjects the manganese was eliminated from the body at an increased rate. Subject W.S. took a smaller oral dose for a shorter period of time. The more rapid elimination in this subject is accounted for by a slight decrease in both the percentage eliminated by the "slow" component, and to a lesser extent, the apparent half-time characterizing the "slow" component. The major effect was the modest increase in the fraction eliminated by the "fast" component accompanied by the much shorter apparent half-time characterizing this component. The apparent half-time of 1.5 days observed is in qualitative agreement with the observation of Britton and Cotzias (5) that the apparent half-time of the "fast" component in mice decreased from about 10 to 2 days as the concentration of manganese in the diet was increased. Once again their study is not strictly comparable because of the differences in experimental design.

In the second study of J.M., there is no evidence for the "slow" component. Thus the same individual who showed the highest percentage of "slow" component with the longest apparent halftime, when manganese intake was low, showed a complete absence of "slow" component when manganese intake was high. This firmly establishes that the retention of manganese in the body is a sensitive function of manganese intake in good agree- 
ment with the point made by Britton and Cotzias (5).

\section{Effect of iron stores on whole body retention of ${ }^{54} \mathrm{MnCl}_{2}$}

The data on S.M., who had no iron in her bone marrow and had an anemia which was corrected by iron, show a definite decrease in the percentage of manganese metabolized by the "slow" pathway, but the apparent half-time characterizing this "slow" component is only slightly decreased as compared to the normal group. Initiation of oral iron therapy at day 50 (400 $\mathrm{mg}$ of iron per day) caused a marked change in the slope of the curve for apparent half-time. Extrapolation of the whole body retention curve to day 80 would have led to the prediction that $10.8 \%$ of the originally injected radioactivity would have remained in the body at day 80 . The observed data showed that, in fact, $15.5 \%$ of the original activity remained at day 80 , i.e., the rate of elimination was decreased by approximately a factor of two after initiation of oral iron therapy. Thus a relationship between iron stores or iron intake and manganese retention is suggested. This interrelationship between iron and manganese metabolism is also implied in a recent study by Alstatt, Pollack, Feldman, Reba, and Crosby (6), which showed a significant increase of manganese concentration in the liver in hemochromatosis. Pallock, George, Reba, Kaufman, and Crosby (7) also presented data in which irondeficient rats absorbed manganese at an increased rate, but the absorption of other nonferrous metals except cobalt was not affected by iron deficiency.

It is interesting that in each instance when the whole body retention curve was experimentally altered 2 months after the injection of radioactivity, a single exponential described the altered disappearance as contrasted with a two component curve in the early part of the study. This suggests that the pool characterized by the "slow" excretory rate did not reversibly exchange with the pool having the fast excretory rate.

The possible interrelationship between the metabolism of iron and manganese, as well as more thorough definition of the "slow" and "fast" pools involved in whole body retention of manganese requires further study. There are a sufficient number of qualitative and quantitative differences between our observations in man and those of Britton and Cotzias (5) in mice to indicate caution in extrapolating details of their data for mice to the situation in man.

\section{Recovery of radioactivity in stools and urine}

The data presented in Table II show that a reasonable recovery was obtained considering the necessity of combining data gathered at widely different counting efficiencies. As reported by Cotzias and Greenough (8), no ${ }^{54} \mathrm{Mn}$ could be detected in the urine even though the experimental arrangement permitted assaying large volumes (nearly $2000 \mathrm{ml}$ for each counting experiment) with unusually high efficiency. Though not shown in Table II, the manganese-loaded subjects also excreted no detectable ${ }^{54} \mathrm{Mn}$ in the urine.

The amount of radioactivity recovered in the stool was in agreement with expectations based upon whole body retention data.

Radioactivity in erythrocytes after intravenous injection of $2.5 \mu \mathrm{c}{ }^{54} \mathrm{MnCl}_{2}$

Injected radiomanganese is rapidly cleared from the plasma and reappears irreversibly bound to red cells, probably as a manganese porphyrin, with the maximum radioactivity in blood occurring 10-20 days after intravenous injection $(9,10)$.

Table III presents data on four of the six subjects of the experiment, only one of whom, W.S., was preloaded with manganese. Again, the advantages of the counting arrangement permitted us to measure even these very small percentages of a quite small quantity of radioactivity with a good degree of confidence and statistical accuracy. The erythrocytes were counted to an average total count of 6500 counts above background.

The data in this portion of the study show that in normal subject (H.M.) $0.15 \%$ of injected ${ }^{54} \mathrm{MnCl}_{2}$ appears in the red cells 17 days after injection. Either manganese deprivation (J.M.), manganese loading (W.S.), or low iron stores (S.M.) reduce the fraction which appears in the blood to about half of this small fraction.

J.M., whose diet was presumably deficient in manganese for the first 60 days of this study, had incorporated $0.064 \%$ of the injected manganese into his red cells by day 18. Massive ingestion of manganese for 25 days caused no decrease in the fraction bound to red cells, although the rate of total body excretion increased, which indicated 
that the ingested manganese did not exchange with red cell manganese pool. Also the originally labeled cells were still in the circulation at day 86 perhaps indicating that a cohort of young cells were were selectively labeled.

S.M., whose iron stores were low, on the other hand, showed a significant decrease in the percentage of manganese bound to her red cells after the oral therapy regimen was initiated. This is shown by the decrease in the fraction on day 86 as compared to day 44 (iron therapy was initiated on day 50). Several mechanisms could account for this decrease. There could have been a dilution effect since her hemoglobin rose from 10.4-12.4 $\mathrm{g} / 100 \mathrm{ml}$ during this interval. If this were the case, an increase in total blood volume would have been required since the same amount of blood $(500 \mathrm{ml})$ was assayed; thus canceling out the effect of increase in red cell volume. Although low blood volumes have been reported in severe anemias, this anemia was so mild that no significant change in blood volume would be expected.

Another possibility is that iron-deficient red blood cells have a decreased survival time and thus the original cells may have been destroyed during the 42 days between assays. The $27 \%$ of the labeled red cells destroyed would not be unreasonable if the ${ }^{54} \mathrm{Mn}$ is incorporated into the red cells independent of the age of the cells and survival time of the cells was essentially normal.

\section{ACKNOWLEDGMENTS}

This investigation was supported by a research grant (HE 06561) from the National Heart Institute, National Institutes of Health, Public Health Service, and, in part, by a grant from the Massachusetts Division, American Cancer Society (No. 952-C-1).

\section{REFERENCES}

1. Borg, D. C., and G. C. Cotzias. 1958. Manganese metabolism in man: rapid exchange of $\mathrm{Mn}^{\mathrm{s}}$ with tissue as demonstrated by blood clearance and liver uptake. J. Clin. Invest. 37: 1269.

2. Recommendations of the International Commission on Radiological Protection ICRP. 1959. Publication 2. Pergamon Press, Inc., New York. 65.

3. Hine, G. J., S. Ginna, and B. A. Burrows. 1961. Geometrical and attenuation corrections for a two crystal whole body counter; body scanning with 8" by $4 " \mathrm{NaI}$ crystal. In Symposium on Radioactivity in Man. 1960. G. R. Meneely and S. M. Linde, editors. Charles $C$ Thomas, Springfield.

4. Cotzias, G. C., D. C. Borg, and A. J. Bertinchamps. 1960. Clinical experiences with manganese. In Metal Binding in Medicine. M. J. Seven and L. A. Johnson, editors. J. B. Lippincott Co., Philadelphia. 50.

5. Britton, A. A., and G. C. Cotzias. 1966. Dependence of manganese turnover on intake. Am. J. Physiol. $211: 203$.

6. Alstatt, L. B., S. Pollack, M. H. Feldman, R. C. Reba, and W. H. Crosby. 1967. Liver manganese in hemochromatosis. Proc. Soc. Exptl. Biol. Med. 124: 353.

7. Pollack, S., J. N. George, R. C. Reba, R. M. Kaufman, and W. H. Crosby. 1965. The absorption of non-ferrous metals in iron deficiency. J. Clin. Invest. 44: 1470 .

8. Cotzias, G. C., and J. J. Greenough. 1958. The high specificity of the manganese pathway through the body. J. Clin. Invest. 37: 1298.

9. Borg, D. C., and G. C. Cotzias. 1958. Incorporation of manganese into erythrocytes as evidence for a manganese porphyrin in man. Nature. 182: 1677.

10. Mahoney, J. P., and K. Sargent. 1967. The plasma disappearance and erythrocyte uptake of ${ }^{54} \mathrm{Mn}$. J. Clin. Invest. 46: 1090. 